\title{
SeqARM: An Association Rule Mining Algorithm Based on Sequence Constraint
}

\author{
Shenshen Bai \\ Department of Information Engineering \\ Lanzhou Vocational Technical College \\ Lanzhou China 730070 \\ shenshen128@sohu.com
}

\begin{abstract}
Sequence constraint mainly considers the occurrence time of item, which can determine whether an association rule is valid. This paper proposes a novel algorithm SeqARM that aims to mine strong association rule with sequence constraint and runs on the second phase of association rule mining. SeqARM employs a fine data structure, named FI-Tree, which is used to save and find frequent itemsets according to a few characteristics of association rule. This work can dramatically reduce the number of invalid association rules, and speed up the procedure of association rules. At last, the experiments prove that SeqARM can improve the performance and effect of the association rule mining.
\end{abstract} Tree

Keywords-association rule; sequence constraint; SeqARM; FI-

\section{INTRODUCTION}

Association rule mining is to find the correlation between items and play an important role in data mining. The procedure of mining association rule includes two phases [5]. The first one is to find all frequent itemsets with a minimum support threshold. And the second one is to generate strong association rules from the frequent itemsets with a minimum confidence threshold. Since [1] proposed this problem, many researchers addressed a lot of works on this issue [2, 8, 6, 9, 3]. However, many presented works focused on the first phase, few authors focused their attentions on the second phase.

In another way, these works did not adequately take the demands of user and the constraints of application into account. The results may not have true values and real meanings. To solve the drawbacks, some researchers proposed constraintbased association rule algorithms [7, 4]. These constraints are directly dependent on the value of items and are easily applied to the process of frequent itemsets mining. However, in realworld applications, there is another constraint which isn't related with the value of item, e.g. the occurrence time of item. Considering the grades of a undergraduate student, obviously the grades of forth semester may not affect the grades of third semester. Let $X=\{C$ Programming $=$ 'A', Data Structure $=$ ' $B$ ', Database $=$ ' $B$ ' $\}$ be a frequent itemset. We know that rule $R 1=\{$ Database $=' B$ ' $=>(C$ Programming $=$ ' $A$ ' $\wedge$ Data Structure $=' B$ ') $\}$ is an illegal association rule, because $C$ Programming and Data Structure are prerequisite courses of Database. However, rule $R 2=\{(C$ Programming $=$ ' $A$ ' $\wedge$ Data Structure $\left.={ }^{\prime} B^{\prime}\right) \Rightarrow$ Database $={ }^{\prime} B$ ' $\}$ is a valid association rule. This constraint cannot be applied to the first phase of association rule mining. We call this kind of constraint as sequence constraint. This paper focuses on sequence constraint and the second phase of association rule mining, proposes a novel strong association rule mining algorithm with sequence constraint, named SeqARM.

The rest of the paper is organized as follows. In section II, the definitions and properties of association rule are presented. Section III proposes the sequence-constraint-based association rules mining algorithm and section IV experimentally shows this algorithm is efficient and effective. Section V summarizes this proposal.

\section{BASIC DEFINITIONS AND PROPERTIES}

The first thing of this section is giving the definitions of frequent itemset and association rule. The second one is presenting two properties of association rule.

Definition 1: Let $I=\left\{I_{1}, I_{2}, \cdots, I_{n}\right\}$ be a set of items, and $D=\left\{T_{1}, T_{2}, \cdots, T_{m}\right\}$ be a transaction database, $T_{i}=\left\{t i d_{i}, X_{i}\right\}$ be a transaction, where $t i d_{i}$ is an identifier and $X_{i} \subseteq I$ is an itemset. The support of an itemset $X_{i}$ is the number of transactions containing $X_{i}$ in $D$, denoted by $\sup (X)$. Given $\varepsilon$ is the minimum support threshold. $X_{i}$ is a frequent itemset if $\left.\sup \left(X_{i}\right) \geq \varepsilon\right)$. If $\left|X_{i}\right|=k, X_{i}$ is called frequent k-itemset.

Definition 2: Given itemset $X_{i} \subset I, X_{j} \subset I$ and $X_{i} \cap X_{j}=$ $\phi, X_{i} \Rightarrow X_{i}$ is an association rule. The support of $X_{i} \Rightarrow X_{i}$ is equal to the support of $X_{i} \cup X_{j}$, i.e. $\sup \left(X_{i} \Rightarrow X_{i}\right)=\sup \left(X_{i} \cup\right.$ $\left.X_{j}\right)$. The confidence of $X_{i} \Rightarrow X_{i}$ is $P\left(X_{j} \mid X_{i}\right)$, denoted by $\operatorname{conf}\left(X_{i} \Rightarrow X_{i}\right), \operatorname{conf}\left(X_{i} \Rightarrow X_{i}\right)=\sup \left(X_{i} \cup X_{j}\right) / \sup \left(X_{i}\right)$.

The basic idea of generating strong association rules from frequent itemset is also "Generation-and-Test" [5]. Given a frequent itemset, the method is (1) firstly enumerating all candidate association rules, (2) then calculating the confidence of each candidate and comparing the confidence with the threshold. However, this process is inefficient, especially for long frequent itemset. Let $L=\{A, B, C\}$ be a frequent 3-itemset, the number of candidate association rules of $L$ is 6 , they are $A=>B C, B=>A C, C=>A B, A B=>C, A C=>B, B C=>A$. For a frequent 4 -itemset, the number of candidates is 14 , and the number of candidates of a frequent n-itemset is $C_{n}^{1}+C_{n}^{2}+$ $\cdots+C_{n}^{n-1}=2^{n}-2$. It can be seen that the second phase also 
play an important role. To improve the performance, the efficient ways are pruning and constraint.

Following are 2 basic properties of association rule, which can be used to effectively prune the candidates that are not strong association rules.

Property 1: Given a frequent $k$-itemset $L_{k}=\left\{I_{1}, I_{2}, \cdots, I_{k}\right\}$, $S \subset L_{k}, S^{\prime} \subset S$, if $S \Rightarrow L_{k}-S$ is not a strong association rule, then $S^{\prime} \Rightarrow L_{k}-S^{\prime}$ is not a strong association rule.

Proof: Let $\lambda$ be the minimum confidence threshold.

$\because S \Rightarrow L_{k}-S$ is not a strong association rule,

$\therefore \operatorname{conf}\left(S \Rightarrow L_{k}-S\right)<\lambda$, i.e. $\frac{\sup \left(L_{k}\right)}{\sup (S)}<\lambda$.

$\because S^{\prime} \subset S$,

$\therefore \sup (S) \leq \sup \left(S^{\prime}\right)$,

$\therefore \frac{\sup \left(L_{k}\right)}{\sup \left(S^{\prime}\right)} \leq \frac{\sup \left(L_{k}\right)}{\sup (S)}$,

$\therefore \operatorname{conf}\left(S^{\prime} \Rightarrow L_{k}-S^{\prime}\right)<\lambda$,

$\therefore S^{\prime} \Rightarrow L_{k}-S^{\prime}$ is not a strong association rule.

Property 2: Given a frequent $k$-itemset $L_{k}=\left\{I_{1}, I_{2}, \cdots, I_{k}\right\}$, $S \subset L_{k}, L \supset L_{k}$, if $S \Rightarrow L_{k}-S$ is not a strong association rule, then $S \Rightarrow L-S$ is not a strong association rule.

Proof: Let $\lambda$ be the minimum confidence threshold.

$\because S \Rightarrow L_{k}-S$ is not a strong association rule,

$\therefore \operatorname{conf}\left(S \Rightarrow L_{k}-S\right)<\lambda$, i.e. $\frac{\sup \left(L_{k}\right)}{\sup (S)}<\lambda$.

$\because L \supset L_{k}$, ,

$\therefore \sup (L) \leq \sup \left(L_{k}\right)$,

$\therefore \frac{\sup (L)}{\sup (S)} \leq \frac{\sup \left(L_{k}\right)}{\sup (S)}$

$\therefore \operatorname{conf}(S \Rightarrow L-S)<\lambda$,

$\therefore S \Rightarrow L-S$ is not a strong association rule.

Applying these two properties into the process of generating the strong association rule can dramatically reduce the number of candidates and improve the speed.

\section{Mining ASSOCIATION RULE WITH SEQUENCE CONSTRAINT}

This section presents the novel sequence-constraint-based association rule mining method. Section III-A proposes the related definitions and section III-B gives a new data structure used to store frequent itemsets. The algorithm of SeqARM is shown in section III-C.

\section{A. Sequence Constraint}

This section presents the formally definitions of sequence constraint.

Definition 3: Let $I_{i}, I_{j}$ be two items, notation $I_{i}>$ $I_{j}$ represents that $I_{i}$ 's occurrence is later than $I_{j}$ 's.
Definition 4: Let $S_{i}, S_{j}$ be two itemsets, if $\exists I_{i} \in S_{i}, \exists I_{j} \in S_{j}$, and $I_{i}>I_{j}$, we denote the relationship of $S_{i}$ and $S_{j}$ as $S_{i}>S_{j}$.

Definition 5: Given itemsets $S_{i}$ and $S_{j}$ satisfy $S_{i}>S_{j}$, if $S_{i}$ is in the left of an association rule, $S_{j}$ cannot be in the right. We name this constraint as sequence constraint.

\section{B. Data Structure}

The major operations of mining strong association rule are generating and searching subsets of frequent itemset. In order to improve the speed of these operations, this work designs an new fine data structure, named FI-Tree (Frequent Itemset Tree), which combines the features of FP-Tree [6] and AFPTree [3]. Next, we will describe FI-Tree through an example.

\begin{tabular}{|c|l|}
\hline tid & items \\
\hline 100 & $\mathrm{I} 1, \mathrm{I} 2, \mathrm{I} 5$ \\
200 & $\mathrm{I} 2, \mathrm{I} 4$ \\
300 & $\mathrm{I} 2, \mathrm{I} 3$ \\
400 & $\mathrm{I} 1, \mathrm{I} 2, \mathrm{I} 4$ \\
500 & $\mathrm{I} 1, \mathrm{I} 3$ \\
600 & $\mathrm{I} 2, \mathrm{I} 3$ \\
700 & $\mathrm{I} 1, \mathrm{I} 3$ \\
800 & $\mathrm{I} 1, \mathrm{I} 2, \mathrm{I} 3, \mathrm{I} 5$ \\
900 & $\mathrm{I} 1, \mathrm{I} 2, \mathrm{I} 3$ \\
\hline
\end{tabular}

Table I

TRANSACTION DATABASE

Table I is a transaction database, which includes 9 transactions and 5 different items [5]. Set the minimum support threshold is 2, mining this database can get 13 frequent itemsets which are shown in table II. The numeric following the ":" is the support.

The algorithm of building FI-Tree is presented in algorithm 1. The input parameter FIs is a set of frequent itemsets, which is in ascending order according to the length of each frequent itemset, if two frequent itemset have the same length; the order is descending according to their supports. And each item in a frequent itemset is descending order according its support. These orders can be seen from table II. Figure 1 depicts the FITree of table II.

\begin{tabular}{|c|l|}
\hline id & frequent itemset \\
\hline 1 & $\{\mathrm{I} 2: 7\}$ \\
2 & $\{\mathrm{I} 1: 6\}$ \\
3 & $\{\mathrm{I} 3: 6\}$ \\
4 & $\{\mathrm{I} 4: 2\}$ \\
5 & $\{\mathrm{I} 5: 2\}$ \\
6 & $\{\mathrm{I} 2, \mathrm{I} 1: 4\}$ \\
7 & $\{\mathrm{I} 2, \mathrm{I} 3: 4\}$ \\
8 & $\{\mathrm{I} 2, \mathrm{I} 4: 2\}$ \\
9 & $\{\mathrm{I} 2, \mathrm{I} 5: 2\}$ \\
10 & $\{\mathrm{I} 1, \mathrm{I} 3: 4\}$ \\
11 & $\{\mathrm{I} 1, \mathrm{I} 5: 2\}$ \\
12 & $\{\mathrm{I} 2, \mathrm{I} 1, \mathrm{I} 3: 2\}$ \\
13 & $\{\mathrm{I} 2, \mathrm{I} 1, \mathrm{I} 5: 2\}$ \\
\multicolumn{2}{|c}{ Table II }
\end{tabular}

FREQUENT ITEMSETS

In FI-Tree, a path $P=\left\{N_{1}, N_{2}, \cdots, N_{k}\right\}$, where $N_{i-1}$ is the parent of $N_{i}, \forall 1<i \leq k$, the parent of $N_{1}$ is root, represents a frequent $k$-itemset whose support is the count of $N_{k}$. It is very 
convenient to get the support of any frequent itemset from FITree by preorder traversal. For example, if we want to search the support of $\left\{\mathrm{I}_{1}, \mathrm{I}_{3}\right\}$, so long as we traverse the shadow node in figure 1, we can get its support, it is 4 .

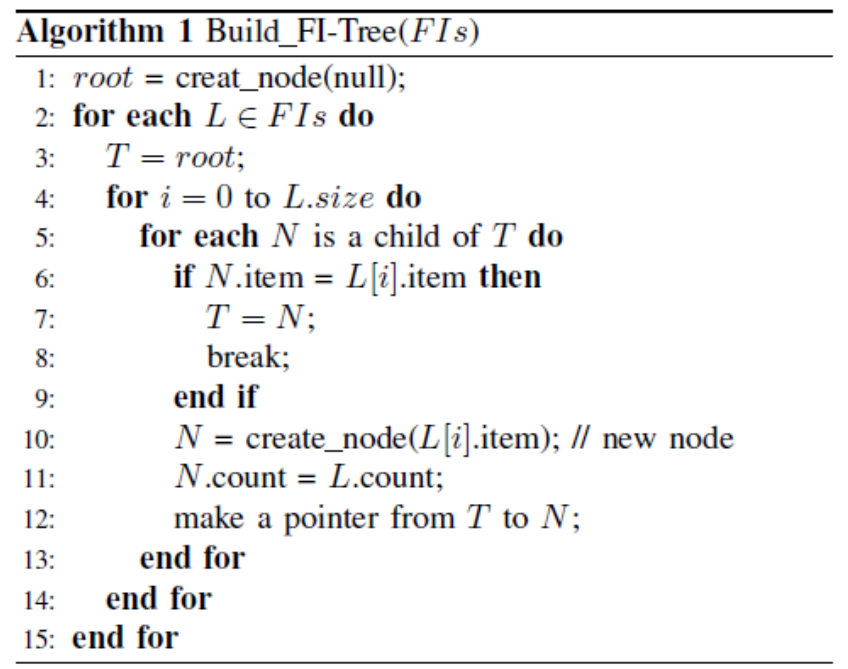

Furthermore, we need an array to store the additive sequence of each item. This constraint doesn't come from the frequent itemset, but come from other external file.

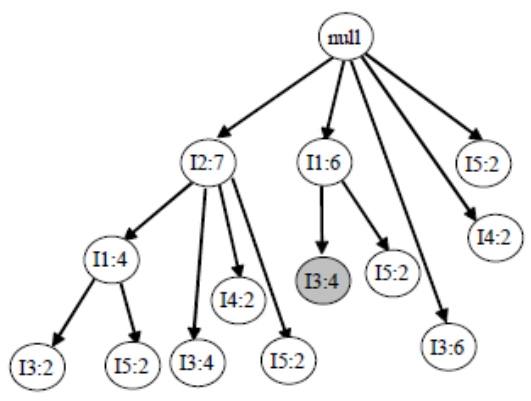

Figure 1. FI-Tree

\section{Algorithm}

This section presents the detail of the SeqARM algorithm. Algorithm 2 is the pseudocode.

At the beginning, SeqARM initialize $R$ with $\phi$ (line 1), which stores the strong association rules. Next, it treats each child of root as a frequent 1-itemset, then calls the iteration function generate_rule() which is the core of SeqARM (lines 2 4). Firstly, generate_rule() visits FI-Tree by preorder traversal. Line 7 adds child node $N$ of current subtree into array $L$. Now $L$ is frequent $(k+1)$-itemset. Line 9 gets all proper subsets of $L$ except $D S$ that saves the deleted itemsets before. The purpose of this step is pruning. Let $L^{i}$ represents $L$ of $i$ th iteration of generate_rule(). According to line 19, $\forall s \in$ $D S, s \Rightarrow L^{i-1}-s$ is not a strong association rule. If $\left|L^{i-1}\right|<$ $\left|L^{i}\right|, L^{i-1} \subset L^{i}$, according property $2, s \Rightarrow L^{i}-s$ is not a strong association rule. Lines 11 21 determine whether $s s \Rightarrow L-s s$ is a strong association rule with sequence constraint where $s s \in S$. If $s s>L-s s$ is true, ss is skipped (lines 12,13).
Otherwise, the confidence of $s s \Rightarrow L-s s$ is calculated (line 15). If $s s \Rightarrow L-s s$ is a strong association rule, it is appended into $R$ (lines 16,17 ). Otherwise, according to property 1 , line 19 deletes $s s$ and its subsets from $S$ and simultaneously appends them into $D S$. If $N$ is not leaf node, generate_rule() is called again (lines 22 24).

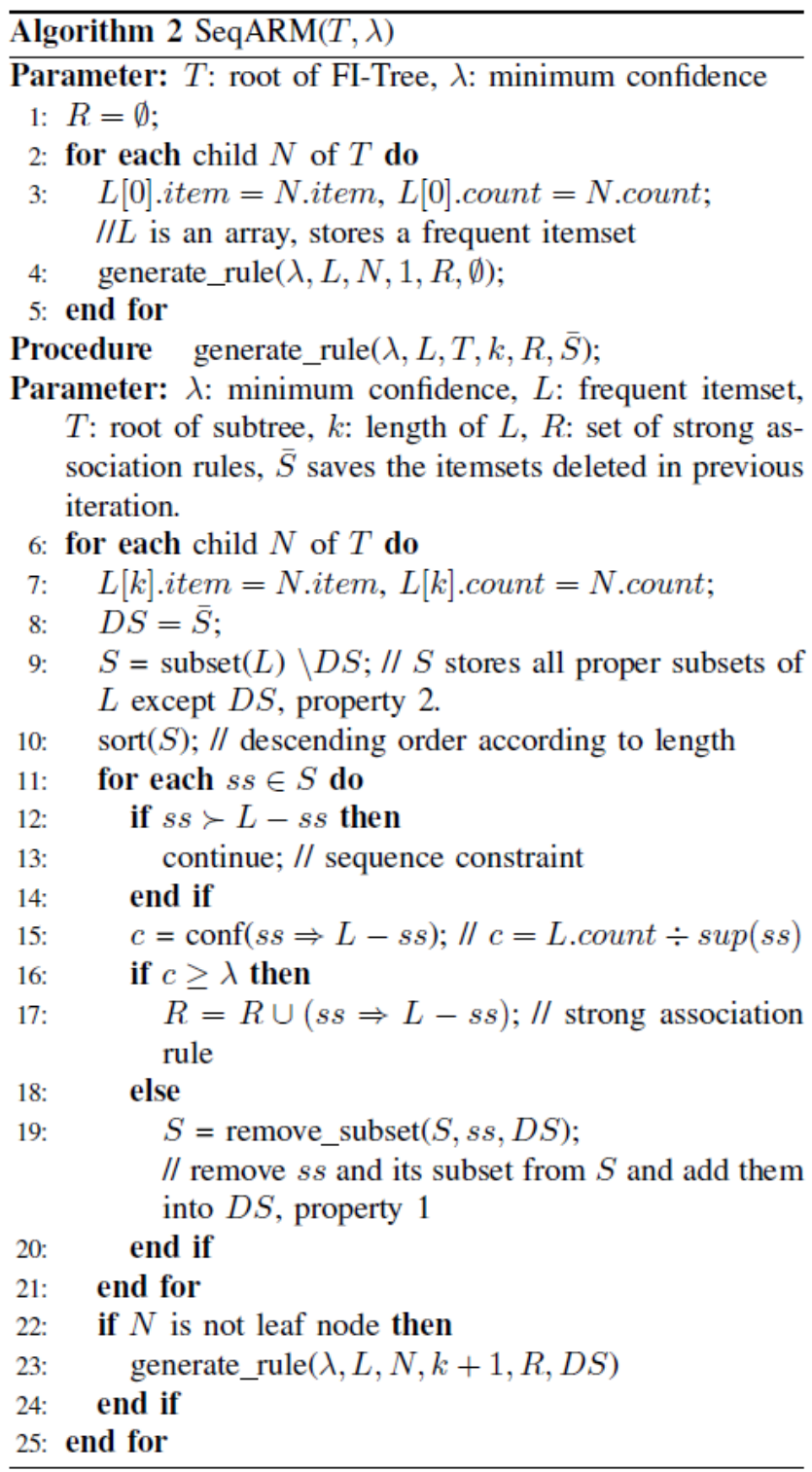

\section{EXPERIMENTAL EVALUATION}

This section experimentally evaluates the effect and performance of SeqARM. The evaluated criteria are running time and the number of association rule. The comparison is traditional association rule method, named as "TradARM". The experiments are conducted on an Intel Core T2080 $1.73 \mathrm{GHz}$ CPU with $1 G$ Bytes of main memory, running on Windows XP professional SP2. All codes are implemented in C\# using Microsoft Visual C\# 2005 Express Edition. We use two real datasets, pumsb* and mushroom, which are standard test datasets and can be downloaded from http://fimi.cs.helsinki.fi/. 
In the experiments we define the sequence between items as following:

Given $I_{i}, I_{j}$ are two items, if $I_{i}>I_{j}$, it is considered $I_{i}$ 's occurrence is later than $I_{j}$ 's, i.e. $I_{i}>I_{j}$.

Figure 2 and 3 show the number of association rule generated by two algorithms. The number of association rule generated by SeqARM is $1 / 2$ to $1 / 3$ of the number generated by TradARM on pumsb*. The number generated by SeqARM is an order of magnitude smaller than the number generated by TradARM on mushroom. From this view, SeqARM can obviously reduce the number of association rule which is invalid, the result is more meaningful.

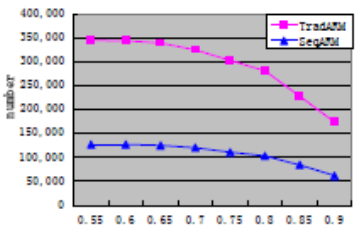

(a) minimum support: 0.45

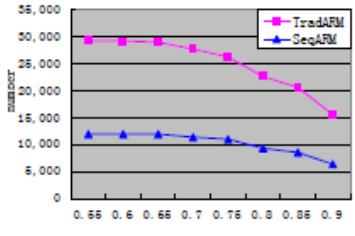

(b) minimum support: 0.5
Figure 2. Number of association rule in pumsb* (a) minimum support: 0.25

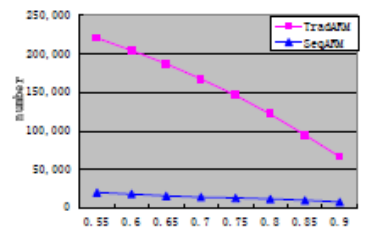

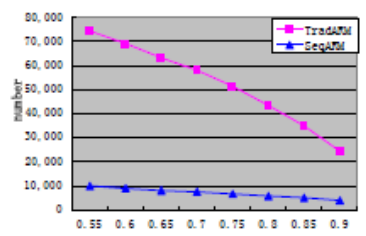

(b) minimum support: 0.3
Figure 3. Number of association rule in mushroom

Figure 4 and 5 give the running time of two algorithms. These two figures demonstrate that SeqARM is faster than TradARM. In figure 4 the time of SeqARM is about half of TradARM's and in figure 5 the time of SeqARM is far less than TradARM. It can be seen from this point that SeqARM is not only able to avoid generating invalid association rule, but also improves the performance of mining association rule.

\section{CONCLUSION}

In this paper, we propose a new kind of constraint of association rule, called sequence constraint. To mine association rule with sequence constraint, a novel algorithm SeqARM is presented. SeqARM uses FI-Tree to store frequent itemsets. Experiments show that SeqARM outperform the traditional method and supply accurate association rules.

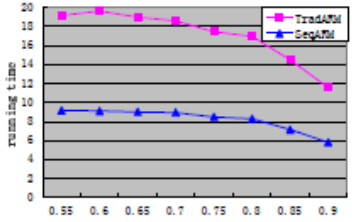

(a) minimum support: 0.45

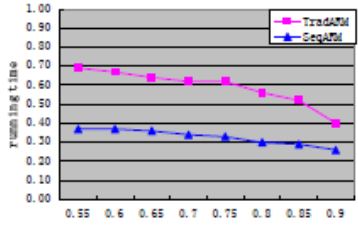

(b) minimum support: 0.5
Figure 4. Running time on pumsb*

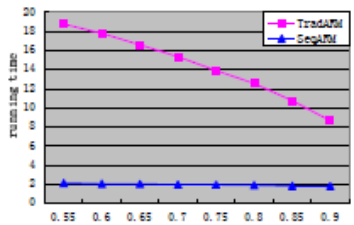

(a) minimum support: 0.25

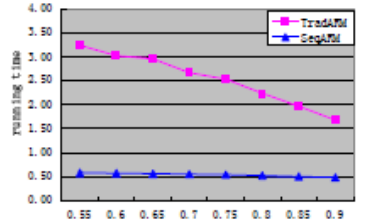

(b) minimum support: 0.3
Figure 5. Running time on mushroom

\section{REFERENCES}

[1] R. Agrawal, T. Imieli'nski, and A. Swami. Mining association rules between sets of items in large databases. In Proceedings of the 1993 ACM SIGMOD international conference on Management of data, SIGMOD '93, pages 207-216, New York, NY, USA, 1993. ACM.

[2] R. Agrawal and R. Srikant. Fast algorithms for mining association rules in large databases. In Proceedings of the 20th International Conference on Very Large Data Bases, VLDB '94, pages 487-499, San Francisco, CA, USA, 1994. Morgan Kaufmann Publishers Inc.

[3] X. Chen, L. Li, Z. Ma, S. Bai, and F. Guo. F-miner: A new frequent itemsets mining algorithm. In ICEBE, pages 466-472, 2006.

[4] L. Cui, S. Yuan, and C. Zhao. Algorithms for mining constrained association rules. Chinese Journal of Computers, 23(02):216-220, 2000.

[5] J. Han and M. Kamber. Data Mining: Concepts and Techniques, Second Edition (The Morgan Kaufmann Series in Data Management Systems). Morgan Kaufmann, 2 edition, Jan. 2006.

[6] J. Han, J. Pei, and Y. Yin. Mining frequent patterns without candidate generation. In SIGMOD Conference, pages 1-12, 2000.

[7] R. Srikant, Q. Vu, and R. Agrawal. Mining association rules with item constraints. In KDD, pages 67-73, 1997.

[8] M. J. Zaki. Scalable algorithms for association mining. IEEE Transactions on Knowledge and Data Engineering, 12:372-390, 2000.

[9] M. J. Zaki and K. Gouda. Fast vertical mining using diffsets. In KDD, pages 326-335, 2003. 